\title{
No idea of risk: Expensive environmental and health panic. Keine Ahnung von Risiko: Teure Umwelt- und Gesundheitspanik.
}

\author{
Authors: Walter Krämer \\ Submitted: $\quad 31$. August 2017 \\ Published: $\quad$ 2. September 2017 \\ Volume: 4 \\ Issue: $\quad 3$ \\ Affiliation: TU Dortmund \\ Languages: German \\ Keywords: Health, Probabilities, Anxiety, Risks, Economy, Environment \\ Language: German Gesundheit, Wahrscheinlichkeiten, Angst, \\ Risiken, Ökonomie, Umwelt, Deutsch \\ Categories: Humanities, Social Sciences and Law \\ DOI: $\quad$ 10.17160/josha.4.3.324
}

\section{Abstract:}

Most people cannot handle risks and probabilities. For alarmists, this is an advantage, for everything new, it is an obstacle. In reality, of course, the health of Germans and people around the globe as measured by life expectancy is constantly increasing. Based on inadequate risk assessments, billions of Euros are wasted every year on unnecessary and even damaging activities. Dr. rer. pol Walter Krämer is a mathematician and economist form the Johannes Gutenberg Universität Mainz, He has worked as a Research Assistant at the Seminar for Statistics at the University of Mannheim; as Assistant Professor at the University of Western Ontario, Canada; Assistant Professor at the Institute for Advanced Studies, Vienna; Professor of Empirical Economic Research at the University of Hanover. He is currently the Spokesman for the DFG Collaborative Research Centre 823: "Nonlinear dynamic models in business and technoloav and a Full Profescor of Fconomic and Social Statictics at the Denartment of Statictics at the

\section{JOSHA Jumna os semene. Humanities and Arts}




\title{
JOSHA Kommentar: Teure Umwelt- und Gesundheitspanik
}

\author{
Kommentar von Roland Mertelsmann und Gerd Steinmann.
}

\begin{abstract}
Most people cannot handle risks and probabilities. For alarmists, this is an advantage, for everything new, it is an obstacle. In reality, of course, the health of Germans and people around the globe as measured by life expectancy is constantly increasing. Based on inadequate risk assessments, billions of Euros are wasted every year on unnecessary and even damaging activities. Dr. rer. pol Walter Krämer is a mathematician and economist form the Johannes Gutenberg Universität Mainz, He has worked as a Research Assistant at the Seminar for Statistics at the University of Mannheim; as Assistant Professor at the University of Western Ontario, Canada; Assistant Professor at the Institute for Advanced Studies, Vienna; Professor of Empirical Economic Research at the University of Hanover. He is currently the Spokesman for the DFG Collaborative Research Centre 823: "Nonlinear dynamic models in business and technology and a Full Professor of Economic and Social Statistics at the Department of Statistics at the University of Dortmund. Den vollständigen Artikel auf Deutsch, in der Frankfurter Allgemeinen Zeitung (FAZ) am 21. August 2017 erschienen, finden Sie im Archiv der Frankfurter Allgemeinen Zeitung (http://www.faz.net/aktuell/finanzen/meine-finanzen/versichern-und-schuetzen/dieumwelt-und-gesundheitspanik-kommt-teuer-15160132.html).

Hier lesen Sie eine kommentierte Kurzfassung des genannten Artikels, dessen Aussagen die Editoren von JOSHA gerne unseren internationalen Lesern zugänglich machen wollen.
\end{abstract}




\section{Zusammenfassung und Kommentar}

$\mathrm{Zu}$ einem Gastbeitrag von Walter Krämer in der Frankfurter Allgemeinen Zeitung am 21.August 2018. Der Autor ist Professor für Wirtschafts- und Sozialstatistik an der TU Dortmund.

\section{http://www.faz.net/aktuell/finanzen/meine-finanzen/versichern-und-}

\section{$\underline{\text { schuetzen/die-umwelt-und-gesundheitspanik-kommt-teuer-15160132.html }}$}

Die meisten Menschen können mit Risiken und Wahrscheinlichkeiten nicht umgehen, was dazu führt, dass fast immer falsche, teure und oft gefährliche Konsequenzen gezogen werden sowohl in der Politik wie im Privaten.

Millionen von Deutschen und Menschen weltweit lassen sich seit Jahrzehnten durch Nichtigkeiten von einer Panik in die nächste treiben: Acrylamid und BSE, Nitrofen und Dioxin, Glycol im Wein, Ehec im Salat und Amalgam in den Zähnen, Nitrat im Grundwasser und Glyphosat im Bier, Vogelgrippe und Schweinepest, Milzbrand, Strahlen und Elektrosmog - um nur einige zu nennen. Dabei nimmt die Lebenserwartung aller Deutschen und auch der Weltbevölkerung ständig zu. Einige der aktuellen Angstmacher heißen Diesel, Feinstaub, Stickoxid usw.. Eine Risikobewertung ist nicht erkennbar, stattdessen wird eine Panik entfacht, viele Bürger geschädigt durch den Wertverlust ihres Autos und eine ganze Industrie in Gefahr gebracht. 


\section{Die Aufregung ist ein Luxus der Wohlstandsgesellschaft}

Man kann sich oft des Eindrucks erwehren, daß die übermäßige Beschäftigung mit „Pseudoproblemen“ auch dazu dienst von wichtigen Herausforderungen und Gefahren abzulenken. Eine alternative, evolutionsbiologische Interpretation wäre, dass Menschen generell Herausforderungen, wie Gefahren brauchen: Wenn es keine echten Gefahren gibt, werden Gefahren erfunden.

Das berühmte Weber-Fechnersche Gesetz aus dem 19. Jahrhundert zeigt, dass bei kleinen Reizen schon kleinste Zuwächse zu Irritationen führen: Je besser die Umweltqualität, desto mehr regt uns ein Staubkorn auf der Windschutzscheibe auf. Die meisten der in unseren Medien zelebrierten Mikro-, Mini- und Midi-Gefahren wären unseren Eltern wie Kleinigkeiten erschienen.

Die Hypersensitivität auch gegen kleinste Risiken des modernen Lebens hat seine Wurzel wohl auch darin, dass es der großen Mehrheit an grundlegenden statistischen Kenntnissen fehlt, mit denen sie kleine oder bedingte Wahrscheinlichkeiten richtig einschätzen könnten. Menschen tun sich schwer damit mit Eintrittswahrscheinlichkeiten für bestimmte Ereignisse richtig umzugehen. Sehr kleinen Wahrscheinlichkeiten wird oft übermäßig viel Beachtung geschenkt, das verzerrt die Handlungen und Reaktionen und führt zu Fehlentscheidungen und inkonsistentem Verhalten. Dringend nötig sind eine verstärkte Ausbildung und Aufklärung schon in den Schulen und in den Universitäten in den Studiengängen aller Fachrichtungen. 


\section{Milliarden gegen nicht-existente Gefahren}

Die hier kritisierte Hypersensitivität für Umwelt- oder Gesundheitsgefahren richtet zudem großen wirtschaftlichen Schaden an. Mit den 1,5 Milliarden Euro, die Verbraucherministerin Renate Künast seinerzeit in großer Hast und ohne viel Überlegung für die Bekämpfung einer in Deutschland nie existierenden BSE-Gefahr aus dem Fenster geworfen hat, hätte sich die Bundesrepublik für ein Jahr eine Universität vom Kaliber Harvard leisten können.

Eine übertriebene Sensitivität für eingebildete Gesundheitsgefahren kann zudem die Gesundheit schädigen. Auch die Asbest-Panik hatte fragwürdige Folgen. Nach Modellrechnungen amerikanischer Statistiker, publiziert in der renommiertesten Wissenschaftszeitschrift „Science“, sind durch die Asbestsanierung und zeitweise Schließung amerikanischer Schulen letztlich mehr Schüler ums Leben gekommen (etwa bei längeren Schulwegen oder im heimischen Swimmingpool), als jemals durch Asbest gestorben wären.

\section{Kosten der Risikophobie}

Die moderne Risikophobie hat auch hohe indirekte Kosten nicht nur durch fehlgeleitete Investitionen sondern auch weil sie Vorbehalte gegen Innovationen und Unbekanntes weckt. Seit dem Farbfernseher trifft insbesondere in Deutschland fast alles Neue, Zukunftsweisende zunächst einmal auf eine große Koalition von Bedenkenträgern. 